\title{
Malignant Mesothelioma Presenting After Resolution of a Seemingly Benign Asbestos Related Pleural Effusion
}

\author{
Authors: \\ Avinash Aujayeb \\ Northumbria Specialist Emergency Care Hospital, Cramlington, UK \\ Correspondence to Avinash.aujayeb@nhct.nhs.uk \\ Disclosure: $\quad$ The author has declared no conflicts of interest. \\ Received: $\quad 10.03 .19$ \\ Accepted: $\quad 27.03 .19$ \\ Keywords: Asbestos, effusion, mesothelioma, pleural. \\ Citation: $\quad$ EMJ Respir. 2019;7[1]:81-85
}

\begin{abstract}
A male patient presented with a signs and symptoms of a respiratory tract infection associated with a pleural effusion. The effusion had negative cytological analysis and a CT scan showed mild pleural thickening. The effusion resolved completely on chest radiographs and the patient was discharged from the respiratory service, but the effusion recurred.

After further investigations and elucidating significant asbestos exposure in the medical history, mesothelioma was eventually diagnosed. The patient is currently receiving chemotherapy with palliative intent.

This paper highlights the importance of ongoing follow-up in a patient with a label of benign asbestosrelated pleural effusion.
\end{abstract}

\section{BACKGROUND}

Asbestos exposure can cause a wide range of respiratory conditions: pleural plaques, benign asbestos pleural effusions, diffuse pleural thickening, malignant mesothelioma, lung cancer, and asbestosis.

Malignant mesothelioma (MM) is a rare form of cancer that usually develops in the pleura or peritoneal serosa. There is a male preponderance, in keeping with occupational exposure in highrisk jobs including production of asbestos sheets, brake, and clutch linings; construction work; shipyard work; or work pertaining to electricians and plumbers. There is a mean latency of about

40 years for pleural MM, and 46 years for the peritoneal subset. Right sided pleural disease is more common, which could be because of the increased pleural surface.

MM carries a poor prognosis; a high index of suspicion, early diagnosis, and treatment is crucial.

\section{CASE PRESENTATION}

A 71-year-old male presented with pleuritic pain, cough, and dyspnoea. He had a background of a renal transplant, gout, and hypertension. $\mathrm{He}$ was on tacrolimus, mycophenolate mofetil, diltiazem, allopurinol, pravastatin, and long-term prednisolone. 
A chest X-ray was performed and showed a right sided pleural effusion (Figure 1a). A chest CT scan (Figure 1b) confirmed the right effusion with mild pleural thickening and small nodules in the inferior aspect of the right parietal pleura.

\section{INVESTIGATIONS}

The patient's white cell count was normal, and C-reactive protein was $97 \mathrm{~g} / \mathrm{L}$, where a normal range is $<5$.

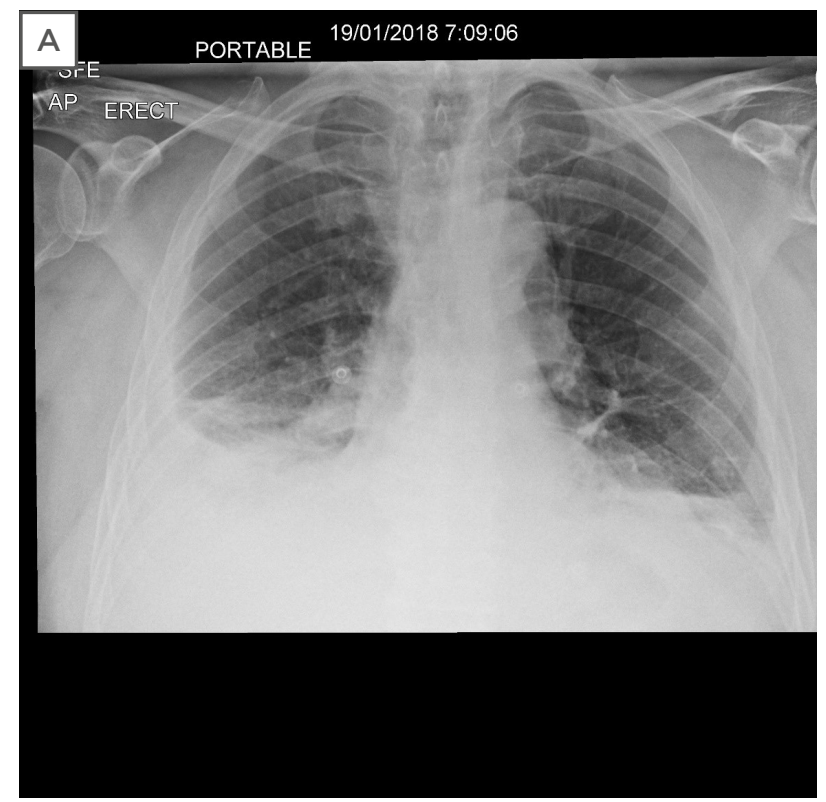

A pleural tap was performed, and $50 \mathrm{~mL}$ of strawcoloured fluid was removed. Analysis showed a protein count of $46 \mathrm{~g} / \mathrm{L}$ (serum total protein count of $76 \mathrm{~g} / \mathrm{L}$ ), lactate dehydrogenase (LDH) of 247 units/L (serum LDH count of 150 units/L), and a pH of 7.55. Cytological and microbiological analyses were negative.

The patient was treated with a 10-day course of co-amoxiclav and clarithromycin for a presumed lower respiratory tract infection and a reactive effusion.

Figure 1: A) chest X-ray showing right pleural effusion; B) CT scan showing right pleural effusion with mild pleural thickening and small nodules and a right pleural plaque.
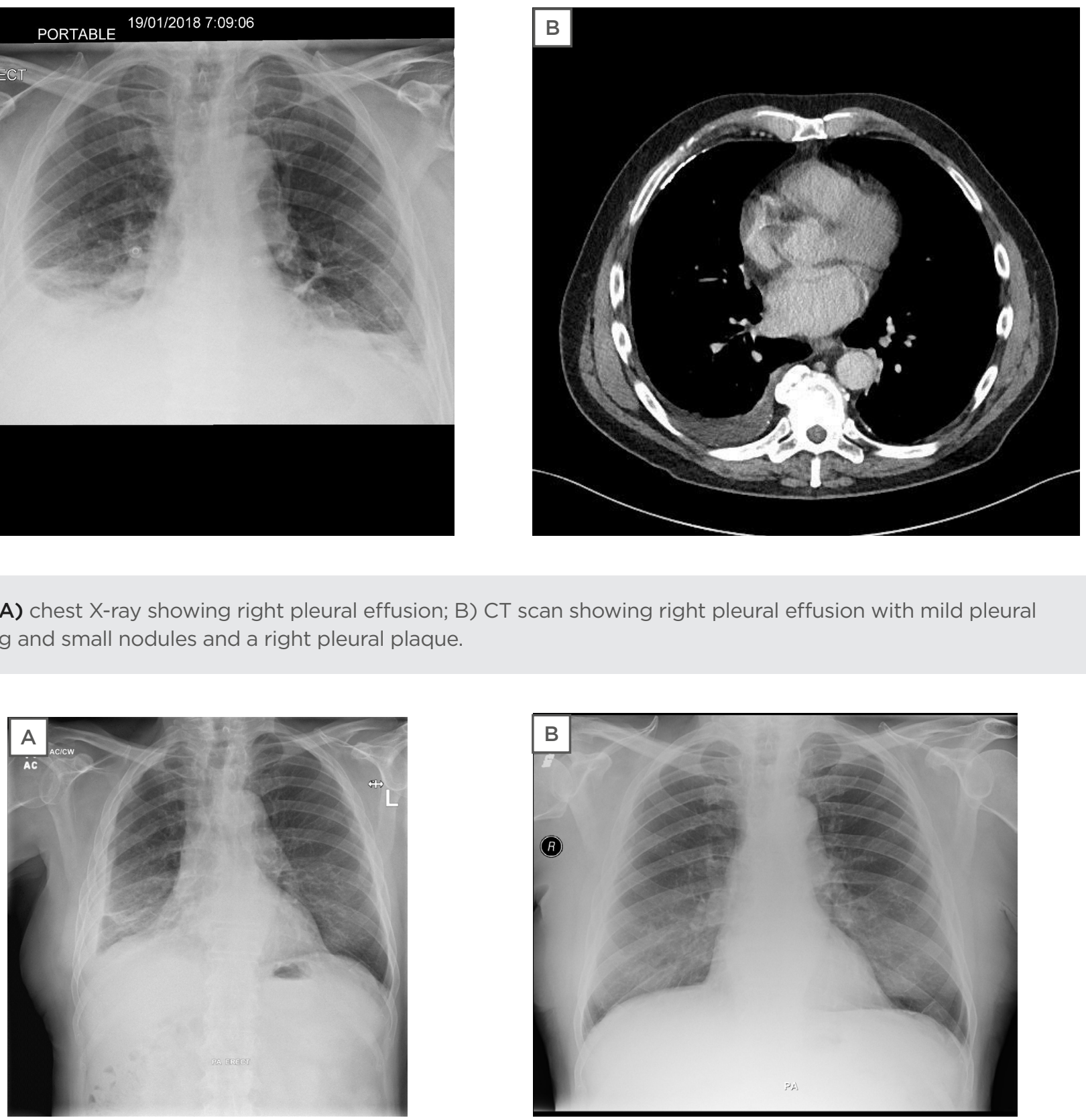

Figure 2: A) chest $X$-ray showing right sided pleural thickening with very small effusion; B) chest $X$-ray showing resolution of right pleural effusion. 
After 2 months, his symptoms had completely resolved. A chest radiograph showed resolution of his effusion (Figure 2b). His C-reactive protein had normalised, and he was consequently discharged from the respiratory service.

When another 3 months had passed, at 5 months after initial presentation, the patient was referred to the pleural clinic with progressive exertional dyspnoea and $2 \mathrm{~kg}$ of weight loss. A repeat chest radiograph showed extensive right sided pleural thickening and a small effusion (Figure 2a). A targeted history revealed that he had never been a smoker. His asbestos exposure started when he was 21 years old and began work as a joiner. He cut asbestos boards and fitted them for roofs, as well as fitting pipes that were lagged with asbestos.

A repeat CT (Figure 3) revealed extensive pleural thickening extending onto the mediastinum with nodular growths which was suspicious for a primary pleural malignancy. An ultrasound-guided pleural biopsy was performed. Histopathological analysis confirmed a sarcomatoid mesothelioma.

\section{DISCUSSION}

A pleural effusion is an exudate if the ratio of pleural fluid protein to serum protein is $>0.5$, ratio of pleural fluid LDH to serum LDH is $>0.6$, or if pleural fluid LDH is greater than two-thirds of the upper limit of normal serum LDH. This is known as Light's criteria. The fluid protein count classifies this effusion as an exudate. ${ }^{1} \mathrm{~A}$ pH of 7.55 is unusual for an exudate. The author suspects there might have been a few air bubbles in the pleural fluid sample, resulting in the reading being impaired.

Cytology of pleural fluid has variable sensitivity; it can be as low as $10 \%$ for MM but increases to approximately $60 \%$ in breast or gynaecological cancers., ${ }^{1,2}$

The patient's symptoms were infective, and the effusion had a negative cytology. There was a sense of false reassurance with the chest radiograph having normalised as seen in Figure 2b. Consequently, the effusion was ascribed to a noncancerous, benign aetiology. ${ }^{3}$ The patient was discharged from the respiratory service. A CT scan was not performed at that time, although a thoracic ultrasound confirmed there was no fluid.
There was no bacterial growth from the pleural fluid and there was no fever, with the caveats that the patient is immunosuppressed, and pleural fluid only yields positive culture in about $40 \%$ of samples. ${ }^{1}$

Relapsing remitting effusions occur with asbestos exposure, and form part of the spectrum of asbestos related pleural disease and benign asbestos pleuritis, although mesothelioma presents with an effusion in roughly $90 \%$ of cases. ${ }^{2}$

The author presumes that the patient had benign asbestos pleural effusion (BAPE) and a lower respiratory tract infection when he first presented. BAPE are usually unilateral and associated with evidence of asbestos exposure, with asbestos plaques in $>90 \%$ of cases. ${ }^{4}$ This is shown in Figure 1b. A typical course of BAPE was followed by the patient. BAPE resolve over an average of 4.5 months and can lead to pleural thickening. A review of the literature suggests that BAPE do not predict an increased risk of mesothelioma beyond that of individuals with similar asbestos exposure without BAPE. ${ }^{4,5}$

However, it is well described in the literature that patients with inflammation of the pleura due to asbestos, or fibrinous pleuritis, have a $15 \%$ risk of developing a pleural malignancy over a mean follow-up time of 16 months. ${ }^{6}$ Two cases of malignant mesothelioma after seemingly benign effusions have been described. ${ }^{7}$ Presence of an asbestos plaque does not prove the absence of a developing malignancy; the mild pleural thickening and nodules seen on the initial CT scan could have meant a tumour was already growing. As such, this case helps to suggest that patients with BAPE require regular follow-up rather than discharge. Chest radiographs can be misleading, even if reported as normal, and a repeat CT scan might have shown more pleural thickening that would have been amenable to a biopsy.

Prognosis with $M M$ is poor and median survival ranges from 8-14 months from diagnosis. There are four main histological sub-types: epithelioid, sarcomatoid, biphasic or mixed, and desmoplastic. The sarcomatoid variant is associated with the most aggressive course, with a median survival of just 4 months. ${ }^{2-4}$

Whilst the diagnosis was delayed here, it is unlikely that an earlier diagnosis would have made a difference as the treatment for $M M$ is chemotherapy only and the patient retained his fitness for treatment. 


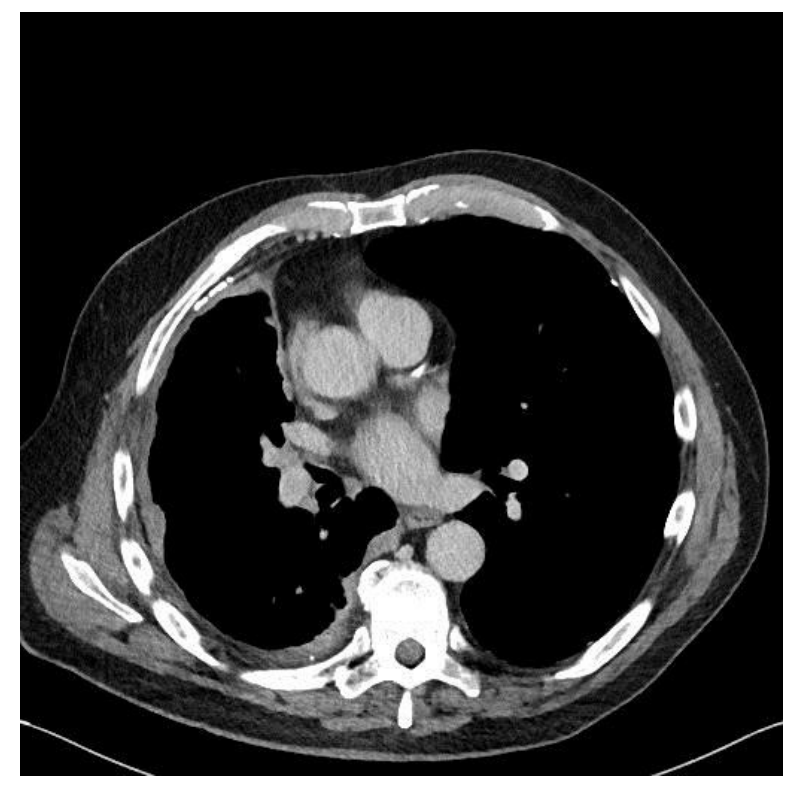

Figure 3: CT scan showing small right effusion with pleural and mediastinal nodularity. A right anterior pleural plaque can be seen also.

\section{OUTCOME AND FOLLOW UP}

The patient has been referred for chemotherapy with palliative intent. The doses of his immunosuppressants have been reduced and he is currently under active follow up.

\section{PATIENT'S PERSPECTIVE}

I had initially felt better on antibiotics and thought that the presentation was of another infection and I was very anxious as I waited on the results of the biopsy. My current concerns are of difficulty sleeping, constant fatigue, and worries about remaining lifespan. I have no interest in usual hobbies, probably due to lack of physical strength, breathlessness, and cough, especially at night

From my wife's perspective, it has been a rollercoaster of emotions from the shock of the diagnosis. We have received excellent care from the medical team, and we hope that the forthcoming chemotherapy treatment will give us some quality of life without many side effects.

\section{TAKE-HOME MESSAGES}

The authors conclude with three take-home messages from this case-study:

> Benign asbestos pleural effusions usually resolve over 4.5 months and are associated with pleural plaques.

> Pleural malignancies, such as malignant mesothelioma, can develop after apparently benign asbestos pleural effusions and in the vicinity of pleural plaques.

> Physicians should have a low threshold for ongoing follow up in patients with seemingly benign pleural effusions and asbestos exposure.

\section{References}

1. British Thoracic Society. BTS Pleural disease guideline 2010. 2010.

Available at: https://www.britthoracic.org.uk/document-library/ clinical-information/pleural-disease/ pleural-disease-guidelines-2010/ pleural-disease-guideline/. Last accessed: 5 March 2019.

2. British Thoracic Society. British
Thoracic Society guideline for the investigation and management of malignant pleural mesothelioma. 2018. Available at: https://www.britthoracic.org.uk/document-library/ 
clinical-information/mesothelioma/ mesothelioma-guideline-2018/btsguideline-for-the-investigation-andmanagement-of-malignant-pleuralmesothelioma/. Last accessed: 5 March 2019.

3. Leung $A N$ et al. $C T$ in differential diagnosis of diffuse pleural disease. AJR Am J Roentgenol. 1990;154(3):487-92.
4. King TE. Asbestos-related pleuropulmonary disease. Available at: https://www. uptodate.com/contents/asbestosrelated-pleuropulmonarydisease\#H1203525457. Last accessed: 5 March 2019.

5. Nobukazu F et al. Clinical investigation of benign asbestos pleural effusion. Pulm Med. 2015;2015:416179.
6. Teng B et al. P240. Outcomes of those diagnosed with chronic fibrinous pleuritis after medical thoracoscopy: A local review. Thorax. 2017;72(3):A214.

7. Muruganandan S et al. Malignant pleural mesothelioma presenting with remitting-relapsing pleural effusions: Report of two cases. Respirol Case Rep. 2018;6(3):e00306. 